Spring Harbor in 1960. By 1970 the theoretical literature contained elaborate electronic mockups, computer simulations and mathematical analyses of such ensembles of mutually synchronizing, nonlinear oscillators. What Enright ably brings to the discussion in 1980 is a detailed computer simulation, complete with noise statistics, built along lines suggested by his concept of pacemaker neurones. His "coupled stochastic systems" model, nicely summarized in Chapter 16, is distinguished from its forerunners by a curious feature: only the longer-period pacers actually synchronize; the others go their merry ways but in so doing provide a pool of potentially entrainable pacers whose contributions become important when the ensemble is exposed to stimuli that affect phases and periods. From this unique feature many of the observed peculiarities of vertebrate circadian rhythms seem to follow, but the extent to which they do so depends on a variety of adjustable quantities in the model. Enright is well aware of the implications of this:

The weary reader may . . . begin to suspect that the model system under consideration shows embryonic symptoms of developing into an amorphous monster: one which can, like an amoeba, engulf and account for any set of data by modification of one parameter or another, but which thereby merely demonstrates its lack of backbone. Can any model with so many parameters lay legitimate claim to being a simple model?

He further argues that explanations that are simple in the sense of elementary physics simply would not be plausible in context of "the workshop of natural selection", which seems more likely to "assemble a reliable pacemaker out of components which are sloppy"

The reader will undoubtedly wonder whether sleep and wakefulness are really the same as rest and activity; whether

\title{
With reference to variable stars
}

\section{R. J. Tayler}

Theory of Stellar Pulsation. By J. P. Cox. Pp. 380. (Princeton University Press: 1980.) Hardback $£ 21.90, \$ 50$; paperback $£ 7.50, \$ 17$.

FOR some time there has been a need for a new book on the theory of variable stars to consider the considerable developments in the subject that have taken place since the publication of Rosseland's The Pulsation Theory of Variable Stars (Oxford University Press, 1949). Now there are two new books: Nonradial Oscillations of Stars (Tokyo University, 1979) by Unno, Osaki, Ando and Shibahashi, which does not discuss the more widely studied and better understood radial oscillations, and the present book which covers the whole subject. J. P. Cox has written many review articles on variable stars and this, the book which has grown out of them, provides a thorough survey both of the foundations of the subject and of recent developments.

In 1949 it was clear that many intrinsic variable stars were pulsating radially, but important questions remained relating to the physical cause of the instability exciting the oscillation, to the observed phase relationships between light and velocity

The British Museum (Natural History), in collaboration with John Wiley, have just published Animal Identification: $A$ Reference Guide in three volumes. Each volume provides a listing of primary reference sources - Vol. 1 to marine and brackish water animals, Vol. 2 to land and freshwater animals (excluding insects), and Vol. 3 to insects - to assist nonspecialists in identifying any animal. The three-volume set costs $\mathfrak{f} 31$. variations, to the processes limiting the instability to finite amplitude and to the evolutionary status of variable stars. The answers to all except the third of these questions are now reasonably clear. However, there is still considerable difficulty in deciding whether a star with a deep convective envelope will pulsate, because there is no good description of time-dependent convection, and there are arguments about whether the stellar masses required to give the correct pulsations agree with those implied by stellar evolution theory. Although there are clues to what produces the finite amplitude limitation of the instability, it is difficult to study the full non-linear problem without crude approximation. The study of nonradial oscillation is much more difficult than that of radial pulsation, but it is clear that non-radial oscillations are present in some stars and, indeed, convection is a nonradial oscillation of very high order. The study of non-radial oscillations has recently been stimulated by observations of small fluctuations in the properties of the Sun.

Cox gives a clear and detailed account of the subject, and this book is a very welcome addition to the literature. If I have any major criticism, it is that it is perhaps too detailed to be used as a textbook; for that purpose I would have omitted some references to topics which could not be discussed in detail. It will, however, be invaluable as a reference book. In particular, it contains a list of relevant papers which is more than 30 pages long. $\square$

$R . J$. Tayler is Professor of Astronomy at the University of Sussex, author of three elementary astronomy textbooks and Managing Editor of Monthly Notices of the Royal Astronomical Society. circadian clocks really do function like pacemaker neurones, but a million times slower; and whether single cells do serve as independent clocks. But Enright's scepticism of his own model leaves little room for innovation by additional critics. Chapters 13 and 14 offer precise predictions for the outcome of feasible experiments which, if not fulfilled, will demand rejection or radical revision of the model. This detailed repartee between observation and interpretation is an edifying grace of Enright's monograph. In it we were treated to a thorough exposition of the present generalizations arising out of circadian physiology; and to a detailed exploration of the consequences of Enright's particular hypothesis.

What then is the upshot? Enright's model, based on observation of rest/ activity cycles in birds and rodents, accounts for many of their peculiarities without having to resort to too many outand-out ad hoc suppositions. It gives a reasonable vision of the kinds of mutual interaction among the pacers that would enhance their precision to the levels sometimes observed.

However, I suspect that much of the fitting to phase-resetting data and periodchanging data could be managed with equal elegance by rather different models; there exists at present little of a factual nature to distinguish alternative visions of the internal organization of the central nervous system clock. Yet here we encounter what, from my present viewpoint among models of human circadian rhythms, seems a primary value of Enright's contribution. Research on sleep/wake rhythms is now in the midst of an explosive blossoming of discovery. Enright's model correlates definite facts and makes distinct critical predictions. This is just what we need, alongside the alternative visions of Wever, Kronauer and others, to catalyse the asking of sharp experimental questions.

Like almost all models in the behavioural sciences, Enright's is partly a fairy tale - in this case, a pleasing one, done with a sense of humour, but not frivolously. He remarks on p. 249 that " . . . there is not the least doubt in my mind that the models as formulated will prove to be wrong; wrong because they are oversimplified; wrong because they have ignored one critical process or another; and wrong because some elements of the models arise from mistaken points of departure". But as Francis Bacon remarks in his Novum Organum, "truth will sooner come from error than from confusion". In my view, debate over Enright's facts and interpretations could take us a long way toward understanding one of the most conspicuous facts of human experience, the rhythm of sleep and wakefulness.

Arthur T. Winfree is Professor of Biology at Purdue University, West Lafayette, Indiana. 